\title{
Regression of diabetic complications by islet transplantation in the rat
}

\author{
A. Remuzzi • R. Cornolti • R. Bianchi • M. Figliuzzi • C. Porretta-Serapiglia • \\ N. Oggioni • V. Carozzi • L. Crippa • F. Avezza • F. Fiordaliso • M. Salio • G. Lauria • \\ R. Lombardi • G. Cavaletti
}

Received: 9 June 2009 / Accepted: 10 August 2009 /Published online: 30 September 2009

(C) Springer-Verlag 2009

\begin{abstract}
Aims/hypothesis Type 1 diabetes is a chronic disease leading to complications such as peripheral neuropathies, nephropathy and cardiovascular disease. Pancreatic islet transplantation is being extensively investigated for blood glucose control in animals and in human type 1 diabetic patients, but the question of whether it can reverse long-term diabetic complications has not been fully explored. We investigated the effects of islet transplantation on diabetic complications in a rat model of streptozotocin-induced diabetes.
\end{abstract}

\footnotetext{
A. Remuzzi $(\bowtie) \cdot$ R. Cornolti $\cdot$ M. Figliuzzi

Department of Biomedical Engineering,

Mario Negri Institute for Pharmacological Research,

Via Gavazzeni 11,

24125 Bergamo, Italy

e-mail: aremuzzi@marionegri.it
}

A. Remuzzi

Department of Industrial Engineering, University of Bergamo, Dalmine, Italy

R. Bianchi · C. Porretta-Serapiglia

Department of Molecular Biochemistry and Pharmacology,

Mario Negri Institute,

Milan, Italy

N. Oggioni $\cdot$ V. Carozzi $\cdot$ L. Crippa $\cdot$ F. Avezza $\cdot$ G. Cavaletti Department of Neuroscience and Biomedical Technologies, University of Milan Bicocca,

Monza, Italy

F. Fiordaliso $\cdot$ M. Salio

Department of Cardiovascular Research, Mario Negri Institute,

Milan, Italy

G. Lauria $\cdot$ R. Lombardi

Neuromuscular Diseases Unit, IRCCS Foundation Carlo Besta, National Neurological Institute,

Milan, Italy
Methods Three groups of rats were used: healthy controls, diabetic and diabetic rats transplanted with microencapsulated islets at 2 months after diabetes induction, when neuropathy was detectable by a decrease in tail nerve conduction velocity $(\mathrm{NCV})$ and impaired nociceptive thresholds. Blood glucose levels and body weight were measured weekly. The variables considered were: thermal (hot plate test) and mechanical sensitivity (Randal-Selitto paw withdrawal test), $\mathrm{NCV}$ and $\mathrm{Na}+, \mathrm{K}+$-ATPase activity in the sciatic nerve. At the end of the experiments hearts were removed for morphometric determination and myocyte number, and kidneys removed for histological examination. Results Islet transplantation in diabetic rats induced normoglycaemia in a few days, accompanied by a rapid rise in body weight and amelioration of impaired nociceptive thresholds, as well as normalisation of $\mathrm{NCV}$ and $\mathrm{Na}^{+}, \mathrm{K}^{+}$ATPase, which were both about $25 \%$ below normal in diabetic rats. Myocyte loss was reduced $(-34 \%)$ by islet transplantation and the observed mild kidney damage of diabetic rats was prevented.

Conclusions/interpretation Besides controlling glycaemia, transplantation of microencapsulated pancreatic islets induced almost complete regression of neuropathy and prevented cardiovascular alterations.

Keywords Cardiovascular disease - Diabetes · Islet transplantation $\cdot$ Nephropathy $\cdot$ Neuropathy

$\begin{array}{ll}\text { Abbreviations } \\ \text { ALT } & \text { Alanine amino transferase } \\ \text { AST } & \text { Aspartate transaminase } \\ \text { BUN } & \text { Blood urea nitrogen } \\ \text { DPN } & \text { Diabetic peripheral neuropathy } \\ \text { IENF } & \text { Intraepidermal nerve fibres } \\ \text { NCV } & \text { Nerve conduction velocity }\end{array}$

Abbreviations

ALT Alanine amino transferase

AST Aspartate transaminase

BUN Blood urea nitrogen

DPN Diabetic peripheral neuropathy

NCV Nerve conduction velocity 
PGP 9.5 Protein-gene product 9.5

TBARS Thiobarbituric acid-reactive substances

\section{Introduction}

As a result of lifestyle changes and ageing of the population, diabetes mellitus is becoming one of the most important worldwide public health problems [1]. It causes multiple organ damage (peripheral nerves, heart, kidney, eyes), leading to an increasing prevalence and severity of several complications [2], among the most frequent of which are peripheral neuropathies [3] and cardiovascular diseases [4].

Diabetic peripheral neuropathy (DPN) occurs in about $34 \%$ of patients who have been hyperglycaemic for more than 15 years [5]. It contributes to morbidity and mortality rates, and severely impairs quality of life, because of paresthesia and pain, the leading cause of non-traumatic amputation [6]. Early disorders of nerve function include slowing in nerve conduction velocity (NCV), followed by axonal degeneration, axoglial disjunction, paranodal demyelisation and loss of fibre density [6]. DPN is mainly a progressive distal axonopathy, with loss of feeling and numbness in feet, hands and legs, which may be accompanied by painful tingling or burning sensations [7]. Diabetic neuropathies are painful in more than $15 \%$ of patients, a substantial proportion of whom complain of chronic pain [6] and poor response to conventional analgesics [8]. Patients often have excessive sensitivity to nociceptive stimuli (hyperalgesia) or may perceive normal stimuli as painful (allodynia) [9].

Diabetic cardiomyopathy, involving structural, metabolic and functional heart damage [10], originates from lesions of coronary arteries and from the effects of high blood glucose levels that can lead to progressive loss of cardiomyocytes by apoptosis, as shown in man [11] or in streptozotocininduced diabetes in rats [12]. Streptozotocin-induced diabetic rats develop cardiomyopathy, with initial impairment of diastolic function followed by left ventricular contractile dysfunction, symptoms that are very similar to the events in type 1 diabetic patients [4].

The streptozotocin-induced diabetic rat shares a number of features with human diabetic neuropathy at the functional and biochemical levels [13]. Decreased NCV, together with reductions in $\mathrm{Na}^{+}, \mathrm{K}^{+}$-ATPase activity, is the hallmark of diabetic neuropathy, but these rats present various types of early neurological dysfunction [14], including altered pain sensation suggesting early involvement of small nociceptive sensory neurons [15]. Alterations in nociceptive threshold can be partially prevented and restored by erythropoietin, prosaposin-derived peptide and neuroactive steroids [14, 16, 17]. Hyperglycaemia plays a major role in the onset and progression of long-term diabetic complications, although insulin deficiency itself may also contribute [18].

Insulin is the standard therapy for patients with type 1 diabetes mellitus. Intensive insulin injections delay the progression of long-term complications [5]. However, this treatment does not induce regression of established tissue damage or of diabetic neuropathy [19]. Moreover, intensive insulin treatment can involve increased risk of lifethreatening hypoglycaemic episodes [5]. An alternative therapy for restoration of endogenous insulin production is pancreas transplantation, which corrects metabolic disorders and reverses nephropathy [20]. However, transplantation bears the risk of potential surgical complications with a 5\% mortality rate at 1 year after surgery [21]. Good metabolic control can be achieved by islet transplantation in the liver circulation, with a minimally invasive approach [22]. Islet transplantation could theoretically be done using immunoisolation devices to avoid the need for immunosuppression [23]. However, the question of whether longterm diabetes complications are halted or reversed by islet transplantation is still not fully known. Studies from other groups have shown that restoration of islet function may improve quality of life, decrease the number of hypoglycaemic episodes and be potentially protective against longterm complications in diabetic patients [24].

We evaluated the effects of immunoisolated islet transplantation on disease complications in streptozotocininduced diabetic rats. The animals were followed up for 60 days after streptozotocin injection to allow development of diabetic complications and were then implanted with syngeneic pancreatic islets. During an additional 60-day follow-up functional measurements were made. At the end of the observation period morphological analysis was done to see whether tissue and organ damage was less severe in transplanted than in non-implanted diabetic animals.

\section{Methods}

Experimental design Inbred male Lewis rats were purchased from Charles River (Charles River, Sulzfeld, Germany) and used as donors and recipients. Animal care and treatment were conducted in conformity with the institutional guidelines, and in compliance with national and international law and policies, e.g. NIH Guide for the Care and Use of Laboratory Animals, US NRC, 1996.

Rats were divided into three groups of eight as follows: healthy control rats, untreated diabetic rats and diabetic rats transplanted with microencapsulated islets at 2 months after diabetes induction. Diabetes was induced by a single injection of streptozotocin $(65 \mathrm{mg} / \mathrm{kg}$ body weight; Sigma, 
St Louis, MO, USA) into the tail vein. Body weight and blood glucose concentration, determined by tail bleeding using strips (Ascensia Elite; Bayer, Basel, Switzerland), were measured weekly. Thermal nociceptive threshold was measured every 20 days. Blood samples were collected at the end of the experiments for haematological determinations (Pentra $60 \mathrm{C}+$; Horiba, Montpellier, France) and for glucose, aspartate transaminase (AST), alanine amino transferase (ALT), creatinine and blood urea nitrogen (BUN) assays (Mira Plus; Horiba). Immediately after killing, the heart, soleus and extensor digitorum longus muscles were carefully dissected out and weighed. The left sciatic nerve, the hind paw footpad skin and a kidney were collected for analysis.

Isolation of pancreatic islets Islets were isolated from the pancreas of Lewis (body weight 250-300 g) rats, using an automatic procedure. Briefly, the pancreases of anaesthetised rats were distended with collagenase $\mathrm{P}$ solution (Boehringer-Mannheim, Mannheim, Germany), removed and then loaded into a digestion chamber at $37^{\circ} \mathrm{C}$. When optimum digestion time was reached, the chamber was flushed with $4^{\circ} \mathrm{C}$ Hanks' balanced salt solution (Gibco InVitrogen, Paisley, UK) and digested tissue purified by centrifugation $(1,500 \mathrm{~g})$ on a Histopaque gradient $(1.077 \mathrm{~g} /$ $\mathrm{ml}$; Sigma). Islets were cultured in a cell incubator in RPMI medium (Gibco InVitrogen), supplemented with fetal bovine serum (EuroClone, Pero, Italy).

Encapsulation and transplantation of pancreatic islets Islet encapsulation was performed as previously described [25]. Briefly, islets were suspended in $1.7 \%$ (wt/vol.) sodium alginate solution (Manugel DMB; Monsanto, Cambridge, UK) at a concentration of 1 islet $/ \mu$. The islet-alginate mixture was then extruded through an air jet droplet generator into a $100 \mathrm{mmol} / 1 \mathrm{CaCl}_{2}$ solution. Gel beads had a diameter of 800 to $950 \mu \mathrm{m}$. The microcapsules containing 3,000 islets were implanted into the rat peritoneal cavity through a small $(1-2 \mathrm{~cm})$ midline incision under tribromoethanol anaesthesia.

Behavioural evaluation The nociceptive threshold to radiant heat was quantified using the hot-plate paw withdrawal test, as previously described [14]. Briefly, a $40 \mathrm{~cm}$ high plexiglas cylinder was suspended over a hot plate and the temperature maintained at $50^{\circ} \mathrm{C}$ to give a latency of about $10 \mathrm{~s}$ for control rats. Paw withdrawal latency was defined as the time between placing the rat on the hot plate and the time when the rat withdrew or began licking the hind paw or manifested discomfort. The mechanical nociceptive threshold was quantified using the Randal-Selitto paw withdrawal test [26] with an analgesy-meter (Ugo Basile, Comerio, Italy), which generates a linearly increasing mechanical force. The results represent the maximum pressure (in grams) tolerated by the animals. The thermal nociceptive threshold response is based on a polysynaptic pathway involving higher centres; the mechanical nociceptive threshold depends on a monosynaptic response. The hot-plate tests were done every 2 weeks, with animals being tested twice and values were averaged. The Randall-Selitto test was done on days 60,80 and 120 .

Nerve conduction velocity in the tail nerve $\mathrm{NCV}$ in the tail was measured by a method described previously [27]. Briefly, recording ring electrodes were placed distally on the tail and stimulating ring electrodes were placed 5 and $10 \mathrm{~cm}$ proximally from the recording point. The latency of the potentials recorded at the two sites after nerve stimulation was determined (peak-to-peak) and NCV was calculated. Measurements were taken in each rat before islet transplantation (day 60) and repeated 80 and 120 days later.

Neuropathology The left sciatic nerve was obtained, fixed by immersion in $4 \%$ (wt/vol.) paraformaldehyde $/ 2 \%(\mathrm{wt} /$ vol.) glutaraldehyde solution for $2 \mathrm{~h}$, post-fixed in $\mathrm{OsO}_{4}$, embedded in epoxy resin and used for light and electron microscopy. Semithin sections were prepared from at least two tissue blocks per animal. The sections were stained with toluidine blue and examined with a light microscope. On the basis of the light microscopic findings, ultrathin sections were prepared from selected tissue blocks, counterstained with uranyl acetate and lead citrate, and examined by transmission electron microscopy (CM 10, FEI; Philips, Eindhoven, the Netherlands).

$\mathrm{Na}^{+}, \mathrm{K}^{+}$-ATPase activity Tibial stumps of the sciatic nerves were collected, desheathed, immediately frozen on dry ice and stored at $-80^{\circ} \mathrm{C}$. For ATPase measurement, nerve specimens were thawed and homogenised in a glass-glass homogeniser (Elvejehm-Potter; DISA, Milan, Italy) at $4^{\circ} \mathrm{C}$ in $0.8 \mathrm{ml}$ chilled solution containing $0.25 \mathrm{~mol} / 1$ sucrose, $1.25 \mathrm{mmol} / \mathrm{l}$ EGTA and $10 \mathrm{mmol} / \mathrm{l}$ Tris, $\mathrm{pH}$ 7.5. Composite, $\mathrm{Na}^{+}, \mathrm{K}^{+}$-ATPase and $\mathrm{Mg}^{++}$ATPase activities were determined spectrophotometrically at $340 \mathrm{~nm}$ (Ultrospec 2100 pro; Amersham-Biosciences, Cambridge, UK) by the coupled-enzyme assay [28], which continuously monitors NADH oxidation. $\mathrm{Na}^{+}, \mathrm{K}^{+}$-ATPase was defined as ouabaininhibitable activity per $3 \mathrm{mmol} / \mathrm{l}$, final concentration. Protein content was determined with a microplate assay protocol (DC Protein; BioRad, Milan, Italy).

Intraepidermal nerve fibre density The density of footpad intraepidermal nerve fibre (IENF) was quantified in three rats per group. Nerve fibres visualised by protein-gene product 9.5 (PGP 9.5) immunohistochemistry of the footpad skin were counted by a method previously 
described [29]. Briefly, hind paw footpad skin was fixed in $2 \%$ (wt/vol.) paraformaldehyde-lysine-periodate for $24 \mathrm{~h}$ at $4^{\circ} \mathrm{C}$ and serially cut into $20 \mu \mathrm{m}$ sections. Three sections were randomly selected and immunostained with polyclonal anti-PGP 9.5 (Biogenesis, Poole, UK). Two blinded observers counted the total IENF in each section using light microscopy at high-power magnification. Individual fibres were counted as they crossed the dermal-epidermal junction. Secondary branching within the epidermis was excluded from the quantification. The length of the epidermis was measured using a computerised system (Microscience, Seattle, WA, USA) and the linear density of IENF (IENF/mm) obtained.

Morphometric determination of myocyte number and volume Number and volume of myocytes were determined on left ventricular sections stained with haematoxylin and eosin. Briefly, the number of myocyte nuclear profiles and the volume fraction of myocytes were counted in an area of tissue section with transversally cut muscle cells. Nuclear length was obtained from 25 measurements in longitudinally oriented myocytes viewed at a magnification of $\times 1,000$ with an Olympus IX51 microscope (Olympus Europe, Hamburg, Germany) equipped with a highcontrast F-VIEW II camera (Olympus Europe). The number of myocyte nuclei per unit volume of myocardium was computed from the number of nuclear profiles divided by the myocyte nuclear length. Myocyte cell volume per nucleus was calculated from the volume fraction of myocytes divided by the number of myocyte nuclei per unit volume of myocardium. The total number of myocyte nuclei in each ventricle was derived from the product of the number per unit volume and the total left ventricular volume. The latter was evaluated by dividing ventricular weight by the specific gravity of muscle tissue.

Kidney histology Kidney was removed, fixed in Bouin's solution (Bio-Optica, Milan, Italy) and embedded in paraffin. Sections $(3.5 \mu \mathrm{m})$ were stained with the periodic acid-Schiff's reagent technique and examined on a Zeiss optical microscope (Jena, Germany). The degree of glomerulosclerosis, tubular dilatation and tubular cast formation were evaluated assigning a score from 0 to 3 ( 0 absent; 1 mild; 2 moderate; 3 severe).

Serum thiobarbituric acid-reactive substances Plasma was collected and EDTA and glutathione were added at 1.34 and $0.65 \mathrm{mmol} / \mathrm{l}$ final concentrations respectively. Thiobarbituric acid-reactive substances (TBARS) levels were determined by modifications of the methods of Yorek et al. [30]. Briefly, $100 \mu \mathrm{l}$ plasma was boiled in $0.6 \mathrm{ml} \mathrm{1 \%}$ (wt/ vol.) phosphoric acid and $0.2 \mathrm{ml}$ thiobarbituric acid $(0.42 \mathrm{mmol} / \mathrm{l})$ for $45 \mathrm{~min}$. The cooled mixture was extracted by agitation with $1.2 \mathrm{ml} n$-butanol and separated by centrifugation (10-20 $\mathrm{min}$ at $1,500 \mathrm{~g}$ ). The upper phase was measured fluorimetrically (Infinite M200; Tecan, Milan, Italy) at excitation wavelength $532 \mathrm{~nm}$ and emission wavelength $553 \mathrm{~nm}$. The calibration curve was prepared with 1,1,3,3-tetraethoxypropane standards 0 to $1.64 \mu \mathrm{mol} /$ $\mathrm{ml}$ final concentration.

Statistical analysis Analysis of variance with comparisons between groups were made using StatView (SAS Institute. Cary, NC, USA). The differences in body weight, NCV, ATPase, TBARS, and myocyte number and volume were analysed by ANOVA and the Tukey-Kramer post test. Data are expressed as mean \pm SEM.

\section{Results}

Body weight and glycaemia Body weight and non-fasting blood glucose concentration were measured weekly during the study and are reported in Fig. 1. Diabetic rats did not grow during the first 60-day observation period. After islet transplantation (day 60), transplanted diabetic rats gained weight, reaching values similar to control rats; the weight of diabetic rats remained significantly lower $(p<0.001)$ (Fig. 1a). After islet transplantation, a steady state of normoglycaemia was obtained in transplanted animals, indicating that transplanted islets were metabolically effective. In untransplanted diabetic animals hyperglycaemia persisted throughout the study (Fig. 1b).

Mechanical and thermal nociceptive thresholds As expected, diabetes was associated with an increase in the thermal withdrawal threshold and a decrease in the mechanical stimulation (allodynia) threshold. Withdrawal latency times of rats during thermal stimulus are reported in Fig. 2a. After streptozotocin injection, the thermal response latency threshold rose progressively, the increase becoming highly significant by day 60 when groups were randomised to no treatment or transplant $(p<0.001)$. Islet transplantation significantly improved the thermal response latency, which almost reached the level of control within 2 weeks (day 76), remaining constant thereafter $(p<0.001)$.

The withdrawal latency time of rats during mechanical stimulus, measured on days 60,80 and 120 is reported in Fig. 2b. As expected, diabetes lowered the mechanical thresholds (46-60\%), but after transplantation they rose significantly from day 80 until day 120 , reaching control values.

Nerve conduction velocity As shown in Fig. 2c, on day 60 $\mathrm{NCV}$ was reduced in diabetic rats compared with controls 

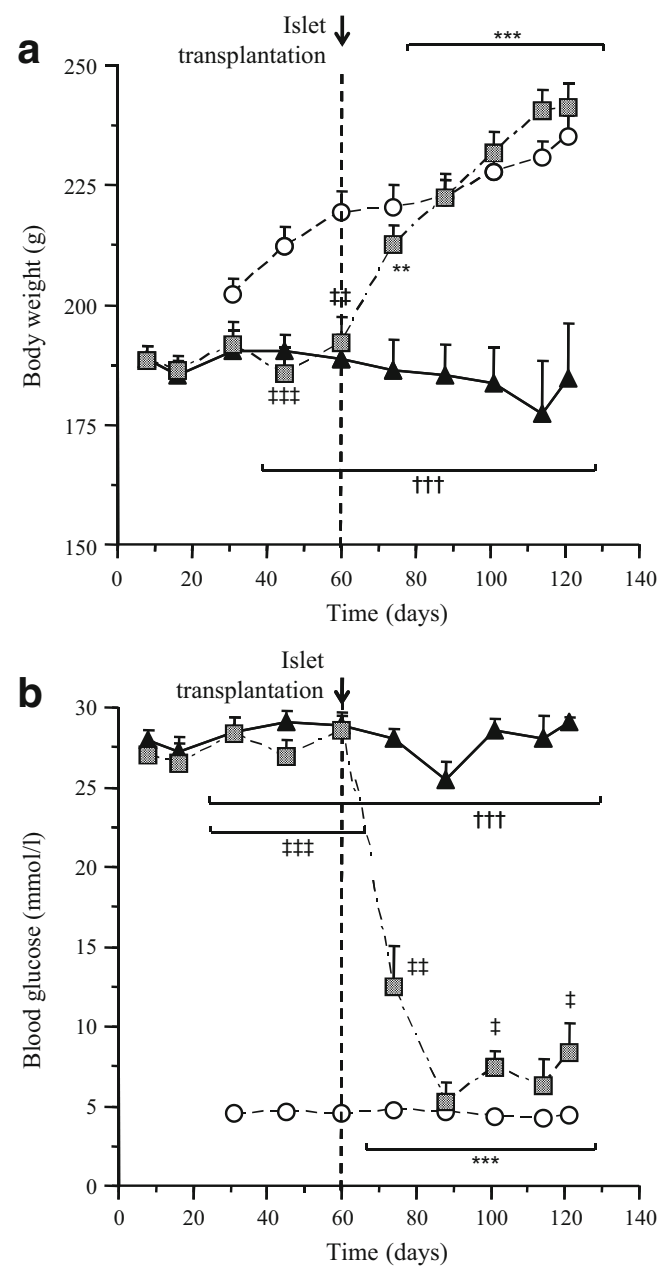

Fig. 1 Body weight (a) and non-fasting blood glucose (b) in the control (white circles) $(n=8)$, diabetic (black triangles) $(n=8)$ and transplanted diabetic (grey squares) $(n=8)$ rats. Islet transplantation was at day 60 . Values are mean \pm SE. $t p<0.05, \pm t p<0.01$, $t+t p<$ 0.001 for transplanted diabetic vs control rats; $* * p<0.01$, *** $p<0.001$ for transplanted diabetic vs diabetic rats; $\dagger \dagger \uparrow p<0.001$ for diabetic vs control rats

$(p<0.001)$. Islet transplantation completely restored the $\mathrm{NCV}$ by day 90 , this effect being maintained until the end of the study.

Sciatic nerve Sciatic nerve specimens obtained at the end of the treatment period were examined. No pathological changes were observed in the diabetic groups under the light microscope. Ultrastructural examination showed mild changes in myelin sheath structure in the largest myelinated fibres, represented by abnormally wide incisures and separation of myelin lamellae, but this was only rarely observed in diabetic rats. The overall mildness of the changes meant no clear effect of islet transplantation was detectable in sciatic nerves of diabetic rats.

$\mathrm{Na}^{+}, \mathrm{K}^{+}$-ATPase activity As expected $\mathrm{Na}^{+}, \mathrm{K}^{+}$-ATPase activity was significantly reduced $(25 \%)$ in sciatic nerve of diabetic rats, but was completely restored by islet transplantation. $\mathrm{Na}^{+}, \mathrm{K}^{+}$-ATPase activity in islet-transplanted diabetic rats and in untreated diabetic rats differed significantly (Table 1).

IENF density IENF mean density averaged $17.68 \pm 0.87$, $14.14 \pm 1.44,16.92 \pm 0.98$ in control, diabetic and transplanted diabetic rats respectively. Although this seemed to suggest an effect of islet transplantation, there was no real difference between the three groups.

Cardiac morphometry Morphometrical evaluation was done to establish: (1) whether the reduced cardiac mass in diabetic rats was due to atrophy or to loss of cardiomyocytes; and (2) whether the increase in cardiac weight in diabetic rats after islet transplantation was related to a reduction in myocyte loss or to hypertrophy. The total number of cardiomyocytes in diabetic rats was significantly lower (34\% less) than in the control group $(p<0.05)$ and significantly greater in transplanted rats than in the diabetic rats $(p<0.05)$ (Fig. 3a). Compensatory hypertrophy led to a $36 \%$ increase of cardiomyocyte volume $(p<0.05)$ in diabetic rats compared with controls. In transplanted rats, cardiomyocyte volume was $13 \%$ less than in the diabetic group (Fig. 3b).

Kidney histology No major structural abnormalities at glomerular level were observed in diabetic animals. Kidneys of diabetic rats had mild tubular dilation and tubular casts. These changes were completely absent in the transplanted diabetic group. In diabetic rats there was an intracellular accumulation of material positive for periodic acid-Schiff's reagent in distal tubules; no such accumulation was seen in islet-transplanted diabetic rats.

Muscle weight Like body weight, muscle weight is affected by diabetes, but the various muscles are affected differently. After 4 months diabetic heart weight was reduced 13\% compared with controls (Table 1), but the difference was not significant. Soleus muscle (a prevalently red-fibre muscle) was less affected than the extensor digitorum longus muscle, a prevalently white-fibre muscle, although both showed a significant reduction in their weight $(17 \%$ and $43 \%$, respectively) (Table 1$)$. Transplanted diabetic rat muscle regained weight similarly to body weight (Table 1).

Serum ALT, AST, creatinine and BUN determination No significant changes were seen in kidney function, which was investigated with serum BUN and creatinine (Table 1). However, ALT was significantly increased in diabetic rats ( $p<0.01$ vs controls), but returned to control values after islet transplantation. AST showed a similar, but not significant pattern (Table 1). 
a

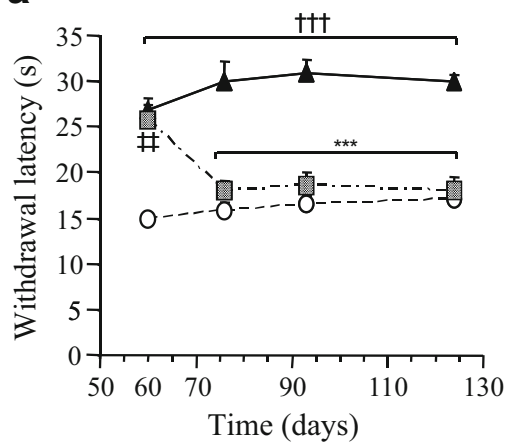

b

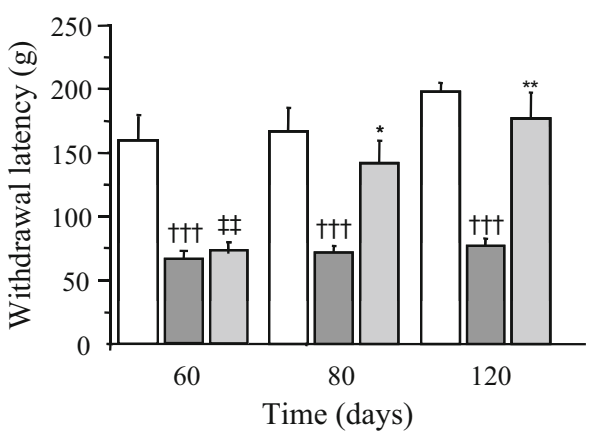

C

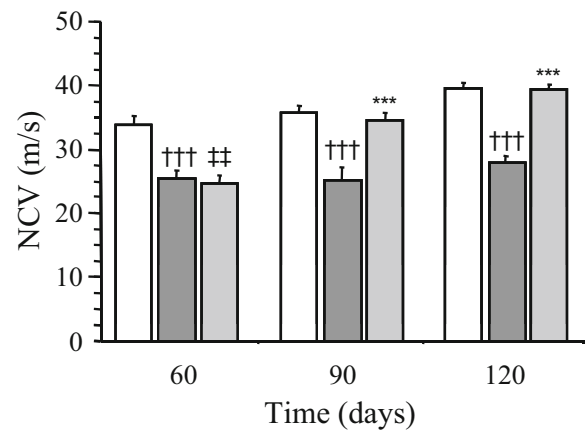

Fig. 2 Thermal nociceptive threshold a in control (white circles) $(n=8)$, diabetic (black triangles) $(n=8)$ and transplanted diabetic (grey squares) $(n=8)$ rats. b Mechanical nociceptive threshold and c NCV in control (white bars) $(n=8)$, diabetic (dark grey bars) $(n=8)$ and transplanted diabetic (light grey bars) $(n=8)$ rats. In mechanical

TBARS To assess the effect of diabetes and islet transplantation on oxidative stress, we measured plasma TBARS. Table 1 shows that diabetes significantly increased TBARS (by $23 \%)$ in plasma $(p<0.05)$, but islet transplantation significantly restored it $(p<0.01)$.

\section{Discussion}

Our investigation was designed to establish whether islet transplantation in streptozotocin-induced diabetic rats could induce regression of diabetic complications. It is well documented [13] that in this model of streptozotocininduced diabetic neuropathy the peripheral nervous system presents evident and reproducible functional, neurophysiological and biochemical changes. Thus, this model is suitable for assessing the chronic effects of therapeutic interventions. Microencapsulated syngeneic islet transplantation in rats with established streptozotocin-induced diabetes achieved satisfactory controlled glucose metabo- nociceptive threshold test at 60 days, $n=4$. Values are mean $\pm \mathrm{SE}$. $\dagger \ddagger p<0.001$ for transplanted diabetic vs control rats; ${ }^{*} p<0.05,{ }^{*} p<$ $0.01, * * * p<0.001$ for transplanted diabetic vs diabetic rats; $\dagger \dagger \dagger p<$ 0.001 for diabetic vs control rats

lism. This model of transplantation with microencapsulated islets would support a strategy for more physiological glycaemic control in allotransplantation studies. After transplantation, there was a significant increase in body weight and a significant reversal of the streptozotocininduced changes in muscle weight, suggesting improvement of the overall metabolic status.

One aim of the present study was to assess whether islet transplantation could relieve neuropathic pain in experimental DPN. Transplantation, in fact, had a significant effect on pain-related behaviour, prolonging withdrawal latencies to paw pressure (mechanical stimuli) and thermal hypoalgesia (hot stimuli). Behavioural tests showed that the thermal and mechanical withdrawal thresholds were early symptoms of DPN, since they occurred 2 months after streptozotocin injection. Our data confirm previous reports that streptozotocin-induced diabetes in rats is associated with mechanical hyperalgesia [14]. This result is clinically relevant because it correlates with reports by diabetic patients [9]. In contrast, conflicting data have been reported about thermal perception in experimental DPN, with
Table 1 Physical and biochemical characteristics of control, diabetic and transplanted diabetic rats

Values are mean $\pm \mathrm{SE}$ of six to ten animals per group

$* p<0.05, * * p<0.01, \quad * * * p<$ 0.001 vs diabetic

$\dagger p<0.05, \dagger \uparrow p<0.01,+\uparrow \uparrow p<$ 0.001 vs control

$\mathrm{Pi}$, inorganic phosphate

\begin{tabular}{llll}
\hline Variables & Control & Diabetic & Transplanted diabetic \\
\hline Tissue weight & & & \\
Heart $(\mathrm{mg})$ & $824 \pm 39$ & $715 \pm 17$ & $870 \pm 34^{*}$ \\
Soleus $(\mathrm{mg})$ & $92.0 \pm 2.9$ & $76.7 \pm 3.3 \dagger \dagger$ & $101.3 \pm 2.3^{* * *}$ \\
Ext. digitorum longus $(\mathrm{mg})$ & $99.0 \pm 2.3$ & $56.7 \pm 3.3 \dagger \dagger \dagger$ & $107.5 \pm 2.5^{* * *}$ \\
Biochemistry & & & \\
TBARS $(\mu \mathrm{g} / \mathrm{ml})$ & $0.19 \pm 0.01$ & $0.24 \pm 0.01 \dagger$ & $0.16 \pm 0.02^{* *}$ \\
ATPase $\left(\mu \mathrm{mol} \mathrm{Pi} \mathrm{h}{ }^{-1}[\mathrm{mg} \text { protein }]^{-1}\right)$ & $0.86 \pm 0.03$ & $0.65 \pm 0.06 \dagger \dagger$ & $0.86 \pm 0.04^{* *}$ \\
BUN $(\mathrm{mmol} / \mathrm{l})$ & $8.15 \pm 0.35$ & $12.22 \pm 0.93$ & $8.17 \pm 0.50$ \\
Creatinine $(\mathrm{mmol} / \mathrm{l})$ & $0.044 \pm 0.004$ & $0.047 \pm 0.004$ & $0.041 \pm 0.004$ \\
ALT $(\mathrm{IU} / \mathrm{l})$ & $78.2 \pm 16.4$ & $191.5 \pm 32.2$ & $94.9 \pm 18.3$ \\
AST $(\mathrm{IU} / \mathrm{l})$ & $157.5 \pm 28.5$ & $282.5 \pm 63.1$ & $178.0 \pm 26.2$ \\
\hline
\end{tabular}



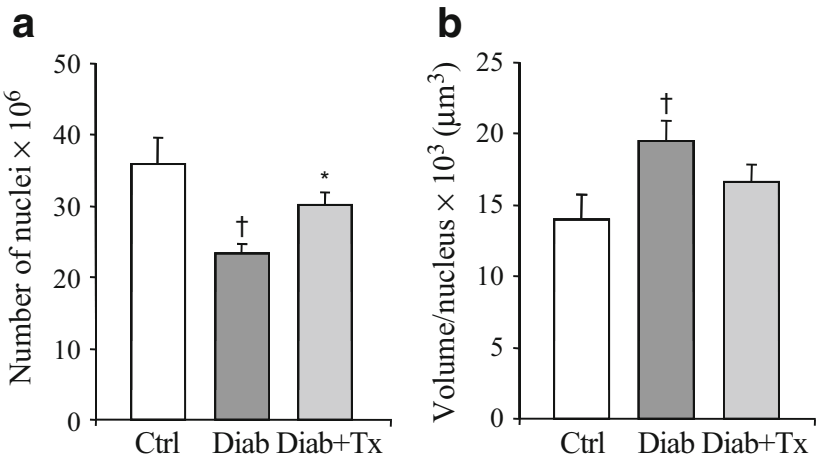

Fig. 3 Total number of myocytes (a) and myocyte volume per nucleus (b) evaluated by morphometrical analysis of heart tissue samples from control (white bars) $(n=8)$, diabetic (dark grey bars) $(n=$ 6 ) and transplanted diabetic (light grey bars) $(n=7)$ rats. Values are mean \pm SE. $* p<0.05$ transplanted diabetic vs diabetic rats; $\uparrow p<0.05$ diabetic vs control rats

different studies showing either an increase (hyperalgesia) [31] or a decrease (hypoalgesia) [14, 32] in the thermal nociceptive threshold in streptozotocin rats. Islet transplantation significantly affected thermal hypoalgesia, with significant reversal of the diabetes-induced mechanical hyperalgesia, which was already normalised 20 days after transplantation.

Our present results show that islet transplantation was able to reverse loss of NCV induced by diabetes. Biochemically, low $\mathrm{Na}^{+}, \mathrm{K}^{+}$-ATPase activity in peripheral nerves offers a potential explanation of NCV slowing during hyperglycaemia. Indeed, we have previously reported that in human nerve specimens the reduction of $\mathrm{Na}^{+}, \mathrm{K}^{+}$-ATPase activity was not simply secondary to fibre loss, but quite possibly contributed to the pathogenesis and self-maintenance of diabetic neuropathy [33]. In diabetic nerves the metabolic derangements result in a decrease in $\mathrm{Na}^{+}, \mathrm{K}^{+}$-ATPase activity, leading to intra-axonal $\mathrm{Na}^{+}$ accumulation and a reduced $\mathrm{Na}^{+}$gradient across the axolemma [34]; insulin replacement can reverse these changes [35]. It has been shown that initiation of intensive insulin treatment significantly improves $\mathrm{NCV}$ in diabetic patients, possibly reflecting restoration of the trans-axonal $\mathrm{Na}^{+}$gradient by strict blood glucose control [36]. $\mathrm{Na}^{+}, \mathrm{K}^{+}-$ ATPase is also sensitive to hypoxia and pharmacological treatments to prevent or restore this activity protect or reverse the drop in NCV [14]. The present study takes these concepts further by demonstrating that islet transplantation significantly counteracted the impairment of $\mathrm{Na}^{+}, \mathrm{K}^{+}$ATPase activity, possibly providing a mechanism that could improve neural function. Besides changes of $\mathrm{Na}^{+}$permeability in the axons, the effect on $\mathrm{Na}^{+}, \mathrm{K}^{+}$-ATPase activity may depend on several mechanisms, such as changes in the subcellular localisation of the enzyme, and in expression or phosphorylation of the different $\mathrm{Na}^{+}, \mathrm{K}^{+}$-ATPase subunits.
Quantification of skin innervating fibre density is the most reliable way to assess small-caliber nerve fibres [37]. Loss of these fibres has been demonstrated in diabetic patients [38] and in streptozotocin-induced diabetic rats and mice $[14,39]$. Since small fibres are closely involved in thermal and nociceptive pathways, while NCV correlates mostly with the impairment of large myelinated fibres, it appears that islet transplantation acts on both populations. The structural nerve fibre damage in proximal peripheral nerves (such as the sciatic nerve) is usually not very severe, and this was confirmed in our experiment. Overall analysis of the peripheral nervous system multimodal assessment suggests that the treatment induced a structural and functional recovery in peripheral nerve activity or at least arrested the progression of neuropathy. These findings have been observed in previous studies by others [40,41] showing that islet transplantation prevented axonal atrophy and the slowing of NCV characterised in rats with streptozotocin-induced diabetes. In contrast, other investigators have reported that islet transplantation was not effective in preventing lesions in the sciatic nerve of rats with sustained normoglycemia [42].

Morphological changes of cardiac tissue were observed in diabetic rats and partially reversed by islet transplantation. Severe myocyte loss and inadequate reactive hypertrophy of the remaining viable myocytes are responsible for the reduction in heart weight in diabetes. Both mechanisms reduced the reserve of the myocardium to sustain increases in mechanical load, influencing the vulnerability to cardiac disease; moreover, in a model of isolated working rat heart, diminishing performance of the diabetic heart was shown to be normalised by islet transplantation through the correction of carbohydrate metabolism defects induced by insulin deficiency [43]. Nearly three of four deaths associated with diabetes are due to cardiovascular complications [44]. Oxidative stress mediated by hyperglycaemia has been suggested in the pathogenesis of several diabetic complications including cardiomyopathy [45]. Among the cardiac alterations, increased myocardial cell death, mainly by apoptosis [46], and hypertrophy are important. We found that re-establishment of the normoglycaemic state in the diabetic rat by islet transplantation attenuated left ventricular myocyte loss (and tended to compensate hypertrophy) to the same extent as antioxidant treatments, which are widely used in clinical practice [47]. This suggests that by reducing oxidative stress, islet transplantation is a powerful means of limiting the development of cardiovascular complications due to chronic hyperglycaemia.

As regards kidney morphology, no major glomerular structural lesions were observed in streptozotocin-induced diabetic rats. In this model, diabetes needs to be maintained for extended periods, usually at least 12 months, in order to detect substantial renal injury [48]. Even at this early stage, 
however, mild damage was observed at the tubular level in diabetic rats. However, after islet transplantation, tubular lesions were completely absent suggesting regression of these early signs of nephropathy.

Our present results clearly show that islet transplantation not only halted the deterioration of neuropathy, but also achieved regression of all diabetes-induced neuropathic alterations within the 2 month follow-up. The most obvious explanation is normalisation of metabolic control, including improvement of glucose and insulin levels, achieved within a few days after the procedure. It is possible that re-establishment of normoglycaemia is not the only mode of action of islet transplantation, and other factors must be taken into consideration. One of the most likely is the beta cell-secreted $\mathrm{C}$ peptide, which can improve sensory nerve function in early-stage type 1 diabetic neuropathy [49] and has renoprotective effects in diabetic nephropathy [50].

Some important issues regarding the treatment of established diabetic complications still need to be addressed, particularly concerning the long-term survival and effect of transplanted islets, and the effectiveness of transplantation in animals with a disease of longer duration, i.e. when more important structural and functional changes are expected. However, the encouraging results of this study offer a sound basis for pre-clinical investigation of islet transplantation in models of diabetes.

In conclusion, our present results show that islet transplantation induces effective regression of established neuropathy and cardiovascular changes associated with diabetes. These findings strongly indicate the need for new strategies focusing on more physiological glycaemic control as a way to arrest the increasing clinical problems posed by the diabetic population worldwide.

Acknowledgements We are indebted to M. Trudu and M. Abbate (Mario Negri Institute, Milan, Italy) for helpful collaboration and renal histology examinations, respectively.

Duality of interest The authors declare that there is no duality of interest associated with this manuscript.

\section{References}

1. Fagot-Campagna A, Bourdel-Marchasson I, Simon D (2005) Burden of diabetes in an aging population: prevalence, incidence, mortality, characteristics and quality of care. Diabetes Metab 31 (Spec No 2):5S35-5S52

2. Gregg EW, Engelgau MM, Narayan V (2002) Complications of diabetes in elderly people. BMJ 325:916-917

3. Said G (2007) Diabetic neuropathy - a review. Nat Clin Pract Neurol 3:331-340

4. Aneja A, Tang WH, Bansilal S, Garcia MJ, Farkouh ME (2008) Diabetic cardiomyopathy: insights into pathogenesis, diagnostic challenges, and therapeutic options. Am J Med 121:748-757
5. DCCT (1993) The effect of intensive treatment of diabetes on the development and progression of long-term complications in insulin-dependent diabetes mellitus. The Diabetes Control and Complications Trial Research Group. N Engl J Med 329:977-986

6. Vinik AI, Park TS, Stansberry KB, Pittenger GL (2000) Diabetic neuropathies. Diabetologia 43:957-973

7. Hoybergs YM, Meert TF (2007) The effect of low-dose insulin on mechanical sensitivity and allodynia in type I diabetes neuropathy. Neurosci Lett 417:149-154

8. McQuay HJ (2002) Neuropathic pain: evidence matters. Eur J Pain 6(Suppl A):11-18

9. Kiguchi S, Imamura T, Ichikawa K, Kojima M (2004) Oxcarbazepine antinociception in animals with inflammatory pain or painful diabetic neuropathy. Clin Exp Pharmacol Physiol 31:5764

10. Fang ZY, Prins JB, Marwick TH (2004) Diabetic cardiomyopathy: evidence, mechanisms, and therapeutic implications. Endocr Rev 25:543-567

11. Frustaci A, Kajstura J, Chimenti C et al (2000) Myocardial cell death in human diabetes. Circ Res 87:1123-1132

12. Fiordaliso F, Bianchi R, Staszewsky L et al (2004) Antioxidant treatment attenuates hyperglycemia-induced cardiomyocyte death in rats. J Mol Cell Cardiol 37:959-968

13. Cameron NE, Cotter MA, Archibald V, Dines KC, Maxfield EK (1994) Anti-oxidant and pro-oxidant effects on nerve conduction velocity, endoneurial blood flow and oxygen tension in non-diabetic and streptozotocin-diabetic rats. Diabetologia 37:449-459

14. Bianchi R, Buyukakilli B, Brines M et al (2004) Erythropoietin both protects from and reverses experimental diabetic neuropathy. Proc Natl Acad Sci U S A 101:823-828

15. Lauria G, Morbin M, Lombardi R et al (2003) Axonal swellings predict the degeneration of epidermal nerve fibers in painful neuropathies. Neurology 61:631-636

16. Calcutt NA, Freshwater JD, O'Brien JS (2000) Protection of sensory function and antihyperalgesic properties of a prosaposin-derived peptide in diabetic rats. Anesthesiology 93:1271-1278

17. Leonelli E, Bianchi R, Cavaletti G et al (2007) Progesterone and its derivatives are neuroprotective agents in experimental diabetic neuropathy: a multimodal analysis. Neuroscience 144:1293-1304

18. Pierson CR, Zhang W, Murakawa Y, Sima AA (2003) Insulin deficiency rather than hyperglycemia accounts for impaired neurotrophic responses and nerve fiber regeneration in type 1 diabetic neuropathy. J Neuropathol Exp Neurol 62:260-271

19. DCCT (1995) The effect of intensive diabetes therapy on the development and progression of neuropathy. The Diabetes Control and Complications Trial Research Group. Ann Intern Med 122:561-568

20. Fioretto P, Steffes MW, Sutherland DE, Goetz FC, Mauer M (1998) Reversal of lesions of diabetic nephropathy after pancreas transplantation. N Engl J Med 339:69-75

21. Gruessner RW, Sutherland DE, Troppmann C et al (1997) The surgical risk of pancreas transplantation in the cyclosporine era: an overview. J Am Coll Surg 185:128-144

22. Shapiro AM, Lakey JR, Ryan EA et al (2000) Islet transplantation in seven patients with type 1 diabetes mellitus using a glucocorticoid-free immunosuppressive regimen. N Engl J Med 343:230-238

23. Kobayashi N (2008) Bioartificial pancreas for the treatment of diabetes. Cell Transplant 17:11-17

24. Fiorina P, Shapiro AM, Ricordi C, Secchi A (2008) The clinical impact of islet transplantation. Am J Transplant 8:1990-1997

25. Figliuzzi M, Plati T, Cornolti R et al (2006) Biocompatibility and function of microencapsulated pancreatic islets. Acta Biomater 2:221-227 
26. Randall LO, Selitto JJ (1957) A method for measurement of analgesic activity on inflamed tissue. Arch Int Pharmacodyn Ther 111:409-419

27. Cavaletti G, Minoia C, Schieppati M, Tredici G (1994) Protective effects of glutathione on cisplatin neurotoxicity in rats. Int $\mathrm{J}$ Radiat Oncol Biol Phys 29:771-776

28. Scharschmidt BF, Keeffe EB, Blankenship NM, Ockner RK (1979) Validation of a recording spectrophotometric method for measurement of membrane-associated $\mathrm{Mg}$ - and NaK-ATPase activity. J Lab Clin Med 93:790-799

29. Lauria G, Sghirlanzoni A, Lombardi R, Pareyson D (2001) Epidermal nerve fiber density in sensory ganglionopathies: clinical and neurophysiologic correlations. Muscle Nerve 24:1034-1039

30. Yorek MA, Coppey LJ, Gellett JS et al (2002) Effect of treatment of diabetic rats with dehydroepiandrosterone on vascular and neural function. Am J Physiol Endocrinol Metab 283:E1067E1075

31. Courteix C, Eschalier A, Lavarenne J (1993) Streptozocin-induced diabetic rats: behavioural evidence for a model of chronic pain. Pain 53:81-88

32. Sugimoto K, Rashid IB, Shoji M, Suda T, Yasujima M (2008) Early changes in insulin receptor signaling and pain sensation in streptozotocin-induced diabetic neuropathy in rats. J Pain 9:237-245

33. Scarpini E, Bianchi R, Moggio M, Sciacco M, Fiori MG, Scarlato G (1993) Decrease of nerve Na+, K(+)-ATPase activity in the pathogenesis of human diabetic neuropathy. J Neurol Sci 120:159-167

34. Brismar T, Sima AA, Greene DA (1987) Reversible and irreversible nodal dysfunction in diabetic neuropathy. Ann Neurol 21:504-507

35. Sima AA (1996) Metabolic alterations of peripheral nerve in diabetes. Semin Neurol 16:129-137

36. Kitano Y, Kuwabara S, Misawa S et al (2004) The acute effects of glycemic control on axonal excitability in human diabetics. Ann Neurol 56:462-467

37. Devigili G, Tugnoli V, Penza P et al (2008) The diagnostic criteria for small fibre neuropathy: from symptoms to neuropathology. Brain 131:1912-1925

38. Hirai A, Yasuda H, Joko M, Maeda T, Kikkawa R (2000) Evaluation of diabetic neuropathy through the quantitation of cutaneous nerves. J Neurol Sci 172:55-62

39. Chattopadhyay M, Walter C, Mata M, Fink DJ (2009) Neuroprotective effect of herpes simplex virus-mediated gene transfer of erythropoietin in hyperglycemic dorsal root ganglion neurons. Brain 132:879-888

40. Schmidt RE, Plurad SB, Olack BJ, Scharp DW (1983) The effect of pancreatic islet transplantation and insulin therapy on experimental diabetic autonomic neuropathy. Diabetes 32:532-540

41. Sima AA, Zhang WX, Tze WJ, Tai J, Nathaniel V (1988) Diabetic neuropathy in STZ-induced diabetic rat and effect of allogeneic islet cell transplantation. Morphometric analysis. Diabetes 37:1129-1136

42. Spadella CT, Machado JL, Caramori CA, Gregorio EA (2002) Successful islet transplantation does not prevent the development of neuropathy in alloxan-induced diabetic rats. Transplant Proc 34:1296-1300

43. Stroedter D, Schmidt T, Bretzel RG, Federlin K (1995) Glucose metabolism and left ventricular dysfunction are normalized by insulin and islet transplantation in mild diabetes in the rat. Acta Diabetol 32:235-243

44. Mourad JJ, Le Jeune S (2008) Blood pressure control, risk factors and cardiovascular prognosis in patients with diabetes: 30 years of progress. J Hypertens 26(Suppl 3):S7-S14

45. Giugliano D, Ceriello A, Paolisso G (1995) Diabetes mellitus, hypertension, and cardiovascular disease: which role for oxidative stress? Metabolism 44:363-368

46. Fiordaliso F, Li B, Latini R et al (2000) Myocyte death in streptozotocin-induced diabetes in rats is angiotensin IIdependent. Lab Invest 80:513-527

47. Tepel M, van der Giet M, Statz M, Jankowski J, Zidek W (2003) The antioxidant acetylcysteine reduces cardiovascular events in patients with end-stage renal failure: a randomized, controlled trial. Circulation 107:992-995

48. Brown J, Sima AA, Tze WJ, Tai J, Thliveris JA (1989) The longterm effect of pancreatic islet allotransplantation on glomerular basement membrane thickening in experimental diabetes. J Diabetes Complications 3:35-39

49. Ekberg K, Brismar T, Johansson BL et al (2007) C-Peptide replacement therapy and sensory nerve function in type 1 diabetic neuropathy. Diabetes Care 30:71-76

50. Hills CE, Al-Rasheed N, Willars GB, Brunskill NJ (2009) Cpeptide reverses TGF-beta1-induced changes in renal proximal tubular cells: implications for treatment of diabetic nephropathy. Am J Physiol Renal Physiol 296:F614-F621 